\title{
Combined low-FOXP3+ and -CD3+tumor infiltrating lymphocytes: a signature of stage II MSS colorectal cancer at high-risk of recurrence
}

\author{
Giuseppe Di Caro ${ }^{1 *}$, Giuseppe Celesti ${ }^{1}$, Fabio Grizzi ${ }^{1}$, Paolo Bianchi ${ }^{1}$, Gianluca Basso ${ }^{1}$, Federica Marchesi ${ }^{2}$, \\ Alberto Mantovani ${ }^{2}$, Alberto Malesci ${ }^{3}$, Luigi Laghi ${ }^{1,3}$
}

From Society for Immunotherapy of Cancer 28th Annual Meeting

National Harbor, MD, USA. 8-10 November 2013

\section{Background}

Low densities of tumor infiltrating lymphocytes (TILs) predict colorectal cancer (CRC) post-surgical recurrence, the likelihood of progression also depending upon tumor stage and microsatellite (MS) status. We benchmarked FoxP3+TIL and CD3+TIL densities as predictor of postsurgical recurrence.

\section{Methods}

FoxP3+ and CD3+TIL densities were measured as the percentage of immune-reactive area (IRA\%) at the tumor invasion front, in a consecutive series of 413 patients (pts) undergone radical surgery for pT3-pT4, stage II $(n=211)$ and III $(n=202)$ CRC, characterized for MS status. Recursive partitioning, as well logistic univariate, interaction, and multivariate analysis were employed for the integrated examination of pathological and immune markers in predicting recurrence.

\section{Results}

In a recursive classification tree, nodal status was the main prognostic factor, discriminating higher recurrence rate for stage III (70/202; 34.7\%; than for stage II (32/ $211,15.2 \% \mathrm{P}<.001)$ pts. Within stage II, $0.23 \%$ FoxP3 + IRA cut-off divided recurrences in $8 / 127(6.3 \%)$ pts with high $(>0.23 \%)$ FoxP3+TILs, and in 24/84 (28.6\%; $\mathrm{P}<.001)$ pts with low $(\leq 0.23 \%)$ FoxP3+TILs. Within the latter group, CD3+IRA\% further branched recurrences, that occurred in $7 / 39(15.2 \%)$ pts with IRA $>1.86 \%$, but in $17 / 38$ (44.7\%; $\mathrm{P}=.003$ ) pts with less IRA\%. Differently, TIL densities did not predict recurrence in stage III.

'Laboratory of Molecular Gastroenterology, Humanitas Clinical and Research Center, Milan, Italy

Full list of author information is available at the end of the article
Interaction analysis revealed that the prognostic effects of FoxP3+and CD3+TIL densities were significantly $(\mathrm{P}<0.05)$ modified by nodal and MS-status status. ROC curve analysis in stage II pts with MSS CRC confirmed the cut-offs identified by recursive partitioning. At multivariate, low $(<0.23 \%$ IRA) FoxP $3+$ and $(<1.86 \%$ IRA) CD3+TILs were both independent recurrence predictors (O.R, 10.22 and 7.85 , respectively; both $\mathrm{P}<.001$ ), and concomitant low-FoxP3+ and -CD3+TILs conferred the highest recurrence risk (O.R. 12.64; $\mathrm{P}<.001)$. Among stage II pts, the low-FoxP3+ and -CD3+TIL signature identified $61 \%(17 / 28)$ of all MSS CRC recurrences, representing $71 \%(17 / 24)$ of post-surgical metastasis (O. R. 18.43; 95\%C.I. 6.68-50.86), while none of 58 pts with high-FoxP3+ and -CD3+TIL experienced recurrences.

\section{Conclusions}

FoxP3+ and CD3+TIL amount, plus tumor MS status efficiently stratify the risk of relapse of stage II CRCs, and may help in tailoring the post-surgical management of patients.

\section{Authors' details \\ 'Laboratory of Molecular Gastroenterology, Humanitas Clinical and Research Center, Milan, Italy. ${ }^{2}$ Department of Immunology and Inflammation, Humanitas Clinical and Research Center, Milan, Italy. ${ }^{3}$ Department of Gastroenterology, Humanitas Clinical and Research Center, Milan, Italy.}

Published: 7 November 2013

\section{doi:10.1186/2051-1426-1-S1-P49}

Cite this article as: Di Caro et al:: Combined low-FOXP3+ and -CD3 +tumor infiltrating lymphocytes: a signature of stage II MSS colorectal cancer at high-risk of recurrence. Journal for ImmunoTherapy of Cancer 2013 1(Suppl 1):P49. 